\title{
Benchmarking phasing software with a whole-genome sequenced cattle pedigree
}

\author{
Claire Oget-Ebrad ${ }^{1 *}$, Naveen Kumar Kadri' ${ }^{2}$, Gabriel Costa Monteiro Moreira' ${ }^{1}$, Latifa Karim³ ${ }^{3}$ Wouter Coppieters ${ }^{3}$, \\ Michel Georges ${ }^{1}$ and Tom Druet ${ }^{1}$
}

\begin{abstract}
Background: Accurate haplotype reconstruction is required in many applications in quantitative and population genomics. Different phasing methods are available but their accuracy must be evaluated for samples with different properties (population structure, marker density, etc.). We herein took advantage of whole-genome sequence data available for a Holstein cattle pedigree containing 264 individuals, including 98 trios, to evaluate several populationbased phasing methods. This data represents a typical example of a livestock population, with low effective population size, high levels of relatedness and long-range linkage disequilibrium.
\end{abstract}

Results: After stringent filtering of our sequence data, we evaluated several population-based phasing programs including one or more versions of AlphaPhase, ShapelT, Beagle, Eagle and Flmpute. To that end we used 98 individuals having both parents sequenced for validation. Their haplotypes reconstructed based on Mendelian segregation rules were considered the gold standard to assess the performance of population-based methods in two scenarios. In the first one, only these 98 individuals were phased, while in the second one, all the 264 sequenced individuals were phased simultaneously, ignoring the pedigree relationships. We assessed phasing accuracy based on switch error counts (SEC) and rates (SER), lengths of correctly phased haplotypes and the probability that there is no phasing error between a pair of SNPs as a function of their distance. For most evaluated metrics or scenarios, the best software was either ShapelT4.1 or Beagle5.2, both methods resulting in particularly high phasing accuracies. For instance, ShapelT4.1 achieved a median SEC of 50 per individual and a mean haplotype block length of $24.1 \mathrm{Mb}$ (scenario 2). These statistics are remarkable since the methods were evaluated with a map of 8,400,000 SNPs, and this corresponds to only one switch error every 40,000 phased informative markers. When more relatives were included in the data (scenario 2), FImpute3.0 reconstructed extremely long segments without errors.

Conclusions: We report extremely high phasing accuracies in a typical livestock sample. ShapelT4.1 and Beagle5.2 proved to be the most accurate, particularly for phasing long segments and in the first scenario. Nevertheless, most tools achieved high accuracy at short distances and would be suitable for applications requiring only local haplotypes.

Keywords: Haplotype, Phasing, Sequencing data, Cattle

*Correspondence: claire.oget@uliege.be

1 Unit of Animal Genomics, GIGA-R and Faculty of Veterinary Medicine,

University of Liège (B34), 4000 Liège, Belgium

Full list of author information is available at the end of the article

\section{Background}

Haplotype phasing consists in the reconstruction of haplotypes inherited from each parent. On autosomes, diploid individuals carry two alleles (eventually identical) at 
polymorphic sites, each allele being inherited from one of the two parents. The combination of alleles from one of the homologous chromosomes is called haplotype, or phase. However, genotyping data obtained with genotyping arrays or from whole-genome sequencing experiments are typically unphased, the origin of each allele remaining unknown. Therefore, statistical phasing methods must be used to determine the set of alleles belonging to each homolog, that were co-inherited.

Haplotype information can be used in many applications in quantitative and population genomics, including missing genotype imputation [1, 2], identification of identical-by-descent (IBD) segments in outbred or experimental populations [3-5], quantitative-trait locus (QTL) mapping [6], haplotype-based association studies [7-9] or genomic predictions [10-12], demographic inference $[13,14]$, identification of signatures of selection $[15,16]$, allele's age estimation [17], estimation of linkage disequilibrium (LD) -based recombination maps [18] or identification of cross-over (CO) events in genotyped pedigrees $[19,20]$. Haplotypes present indeed higher LD with underlying causative variants and allow the estimation of the length of shared IBD segments, a measure related to the number of generations to their common ancestor [21]. Haplotypic information is also required to understand interactions among tightly linked loci, for instance to study allele specific expression, identify deleterious compound heterozygotes or to determine how combinations of variants affect gene expression [22, 23].

Haplotype phasing methods can be divided into two main groups (as reviewed in Browning and Browning [24]): those relying on pedigree relationships (e.g., [25, 26]) and those that can be applied in samples of unrelated individuals by exploiting LD information, often referred to as population-based methods (e.g., [7, 27, 28]). Nevertheless, some methods present hybrid properties by exploiting both sources of information (e.g., [29-31]). Other methods apply heuristic rules by matching target haplotypes to libraries of reference haplotypes in windows (e.g., [30]). Long-range phasing methods use such heuristic approaches and rely also on the identification of surrogate parents [29,32]. Most recent advances in phasing methods were related to their ability to handle huge data sets, including thousands of sequenced samples [3335]. Phasing accuracy of these different approaches will impact the outcome of different haplotype-based applications and must be assessed. Although this is most often initially tested in human populations, it should ideally be realized in populations with different demographic histories and levels of relatedness.

Here we take advantage of a unique sequenced cattle pedigree to assess accuracy of several population-based phasing methods, including recent methods commonly used in livestock species. This sample is a typical example of a livestock population with reduced effective population size, high levels of relatedness and long-range LD, and containing 100 to 200 sequenced individuals. We show that such data can be phased with extremely high accuracy. In addition, we illustrate that the raw sequence data requires stringent filtering to obtain accurate haplotypes.

\section{Results}

Quality of whole-genome sequence genotype data after applications of different procedures

We assessed the quality of the genotyping data by comparing the number of identified $\mathrm{CO}$ with the evaluated data to the expected number of $\mathrm{CO}$, obtained with a high-quality reference map validated with 115,967 genotyped individuals and 30,331 SNPs (see Methods). In both cases, $\mathrm{CO}$ were identified using a pedigree-based approach [36]. The ARS-UCD1.2 bovine genome assembly [37] was used as starting point for the reference map. A total of 18 SNPs showing evidence of incorrect map positions were then discarded. Among those, 13 matched with regions also flagged by Quanbari and Wittenburg [38] as potential errors in the genome build. After removal of these SNPs, we found no more evidence for map errors. Using this reference map and our sequenced pedigree containing 264 individuals, we found an average of $24 \mathrm{CO}$ per individual, 26 and 23 in males and females, respectively.

When CO were estimated using the 15,327,429 SNPs that passed the variant quality score recalibration (VQSR) procedure (with the threshold set to 99.9), the average counts per meiosis were highly inflated, equal to 1416 (Fig. 1). When the threshold for the VQSR filtering was set to 97.5, resulting in the selection of $11,030,905$ SNPs, the average number of $\mathrm{CO}$ dropped to 254 confirming that the data quality was improved. However, this value was 10 times larger than the expected values, clearly indicating that the sequence genotype data required further cleaning. Subsequent selection of a subset of $8,435,899$ variants behaving like true Mendelian variants (see Meth$o d s$ ), with genotype frequencies close to Hardy-Weinberg proportions, and with minor allele frequency higher than 0.01 , resulted in the identification of $167 \mathrm{CO}$ on average per meiosis, still six times above expectations. Refining genotype calls using Beagle4.1 [39] clearly improved the genotype quality, the number of identified $\mathrm{CO}$ being reduced by a factor 3 (51 CO per meiosis on average). We then removed regions presenting high coverage, excessive levels of recombination or of genotyping errors (see Methods), resulting in the removal of 18,220 additional SNPs. The total number of SNPs was then $8,417,679$ ranging from 166,292 (chromosome 25) to 529,626 


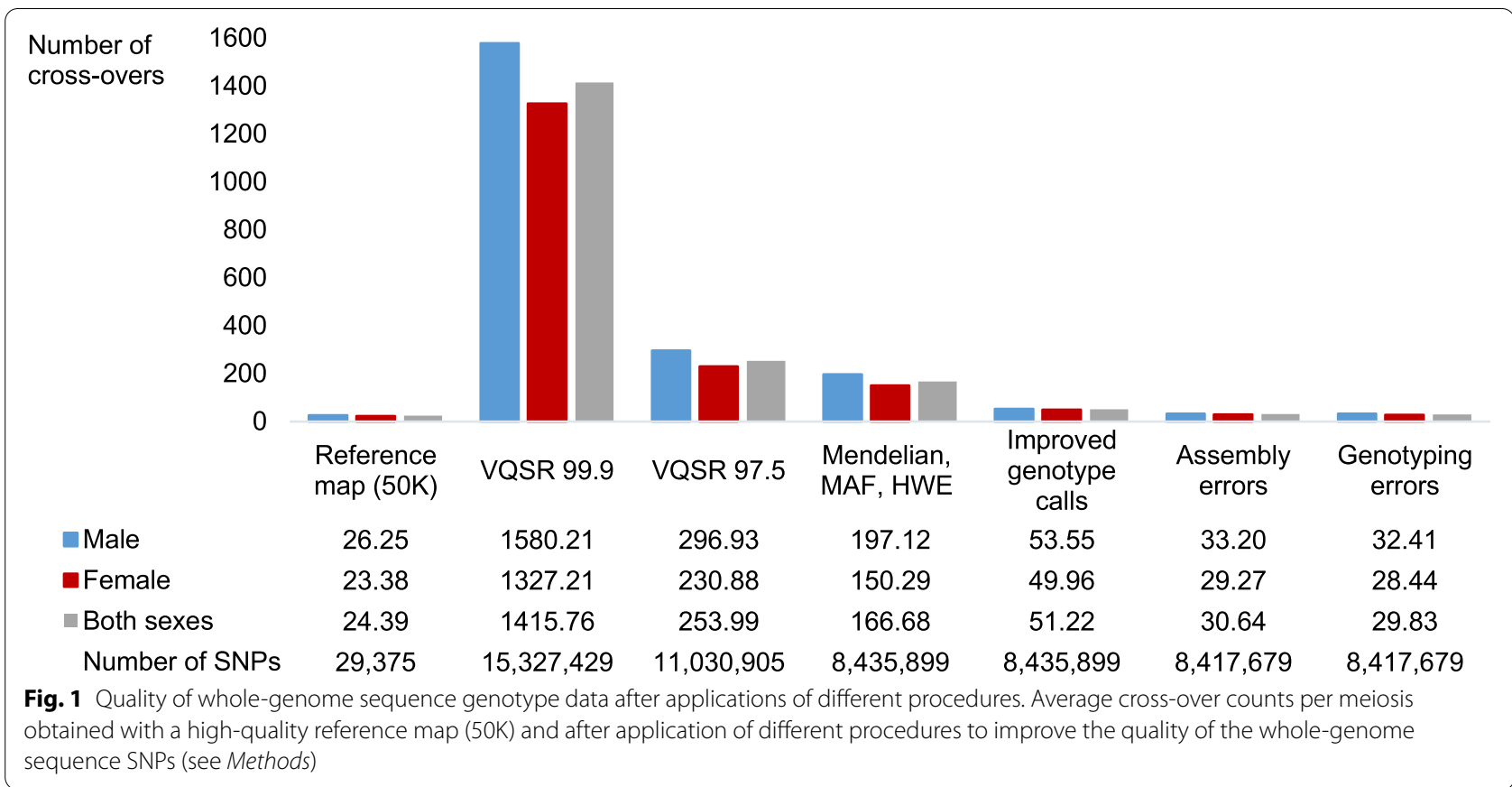

(chromosome 1) per chromosome (Table S1 in Additional file 1). This removal further improved the quality of our data as the number of identified $\mathrm{CO}$ dropped to 31 $\mathrm{CO}$ per meiosis on average. Finally, by setting remaining genotypes that were discordant in parent-offspring pairs to missing (e.g., opposite homozygous), the average number of identified CO was further reduced to $30 \mathrm{CO}$. Overall, the application of these different procedures allowed us to reduce the average number of $\mathrm{CO}$ from 1416 to 30 $\mathrm{CO}$, closer to expectations. Nevertheless, we still identified on average 5 additional $\mathrm{CO}$ with the final sequence data compared to results obtained with the high-quality reference map. These 5 additional $\mathrm{CO}$ might be missed with the 50K map but could also correspond to errors, meaning that haplotypes obtained with a family-based approach might still contain a few errors.

\section{Comparison of phasing quality achieved with different population-based phasing methods \\ Strategy}

To assess the phasing quality from different LD-based methods we used the haplotypes from 98 sequenced individuals that had both their parents also sequenced (sequenced trios). The haplotypes of these sequenced offspring (validation individuals) were phased using Mendelian rules, that are exact in absence of genotyping errors, to serve as the "true haplotypes". Populationbased approaches were then applied in two scenarios, either with only the 98 validation individuals (scenario 1), or with the full data set consisting in 264 individuals, but ignoring the pedigree relationships (scenario 2). Most phasing metrics are computed with respect to heterozygous markers phased in the true haplotypes (the gold standard) with Mendelian rules since these markers are informative. On average, each of the 98 individuals had $1,964,220$ such informative markers in both scenarios (Table S1 in Additional file 1). The different metrics used to assess phasing quality are described in the Methods section and most of them are illustrated in Fig. 2.

\section{Phasing yield}

Most of the tested phasing methods achieved 100\% phasing yield, and this was almost true also for FImpute3.0 that phased on average more than $99.99 \%$ of the heterozygous SNPs in both tested scenarios. Only AlphaPhase1.3 failed to phase all the SNPs, with on average 97.5 and $98.7 \%$ of phasing yield in the first and in the second scenarios respectively.

\section{Switch error count (SEC) and rate (SER)}

Median switch error count (SEC) and rate (SER) computed on the 98 validation individuals are provided for the main phasing algorithms and each scenario in Fig. 3 and Table 1 . When only the 98 validation individuals were used for phasing, the median SEC was around 4500 to 5000 for a group of methods including AlphaPhase1.3, Eagle2.4 and FImpute3.0. These values correspond to a SER slightly below $0.25 \%$, meaning that switch errors occur on average every 400 informative markers. Haplotypes obtained with Beagle4.1 had clearly lower SEC than 


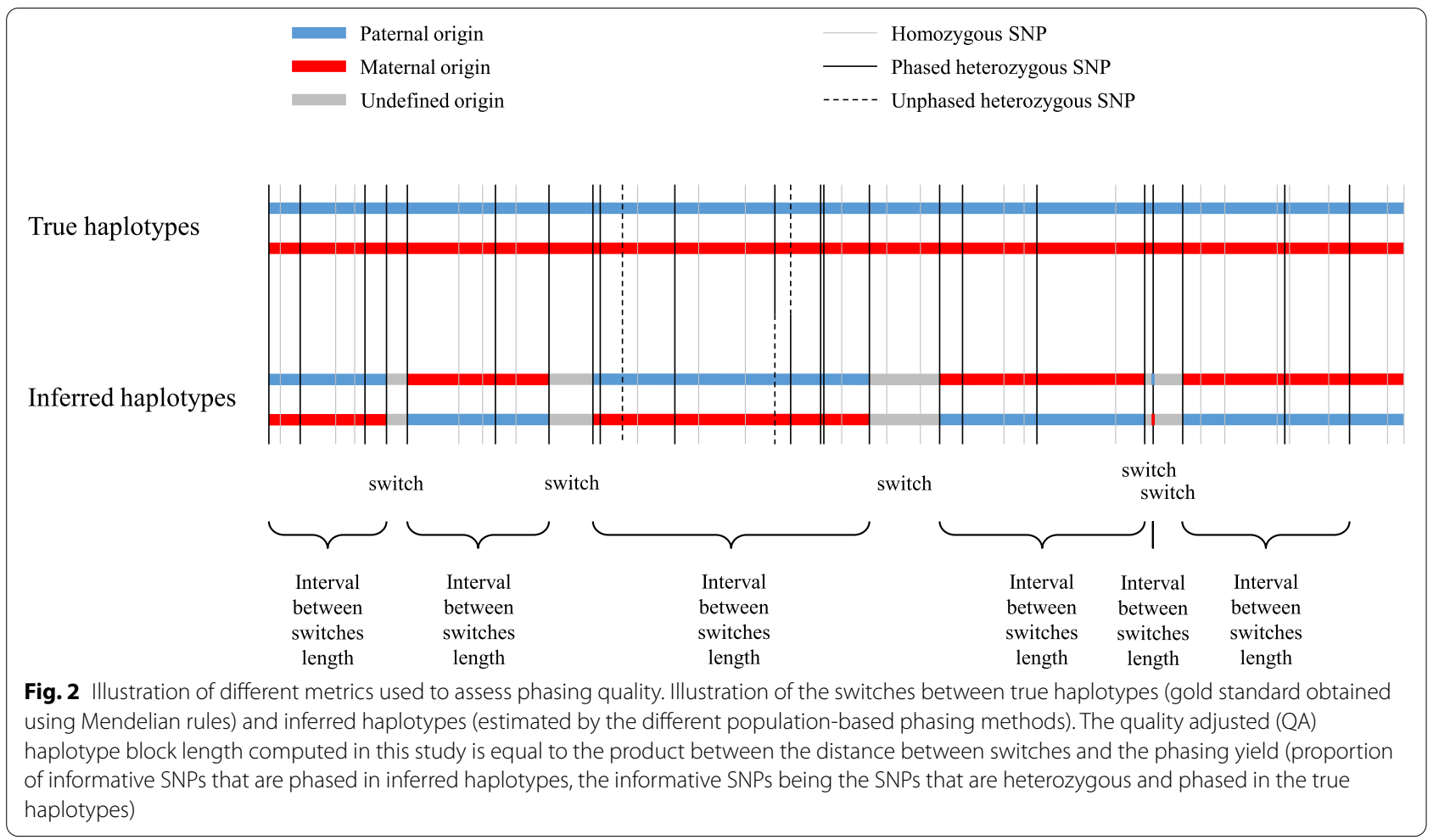

these first methods, close to 2750. ShapeIT4.1 performed even better with median SEC and SER below 400 and $0.02 \%$ respectively, corresponding to one switch error every 5000 informative markers. Finally, Beagle5.2, relying on a new algorithm compared to earlier Beagle versions (until Beagle4.1), resulted in the lowest SEC and SER, outperforming all other methods, the median values were below 200 and $0.01 \%$ per individual, corresponding to one switch every 10,000 informative markers. This represents a reduction by a factor 20 compared to AlphaPhase1.3, Eagle2.4 and FImpute3.0, whereas ShapeIT4.1 generated ten times fewer switches than these methods. When methods are compared with other summary statistics related to SEC and SER such as the mean, minimal and maximal values or as their range (Fig. 3A and Tables S2-S3 in Additional file 1), the ranking of the method remained similar with Beagle5.2 performing best, followed by ShapeIT4.1. When comparing different versions of the same software in terms of SEC and SER (Figure S1A in Additional file 1), we observed that newer versions are more accurate as expected. In particular, AlphaPhase1.3 represents a major improvement with respect to AlphaPhase1.1. Regarding Beagle's versions relying on a directed acyclic graph, Beagle3.3 and Beagle4.0 had close performances and Beagle4.1 appeared as an important improvement. Several of these versions of Beagle presented a lot of variation among individuals.
Regarding the latest Beagle's versions, relying on the $\mathrm{Li}$ and Stephens model [40], Beagle5.1 was slightly better than Beagle5.0 whereas Beagle5.2 represented a substantial improvement. Finally, ShapeIT2 and ShapeIT4.1 achieved similar performances.

When a larger data set was used for phasing, consisting in 264 individuals including the sequenced parents (scenario 2), the phasing accuracy improved for most of the methods (Fig. 3B and Table 1), with less variation among individuals. For AlphaPhase1.3, the SEC reduction remained however modest and it consequently ranked last. For all the other phasing methods, the median SEC was below 1000, around 900 and 350 for Eagle2.4 and Beagle4.1, respectively. FImpute3.0 showed the highest improvement compared to scenario 1, the median SEC being reduced by almost 40 folds and dropping to 115 . However, Beagle5.2 and ShapeIT4.1 still performed best with median SEC values equal to 55 and 50 , respectively. These values correspond to extremely low median SER, equal to 0.0027 and $0.0026 \%$, respectively, and to one switch error every 40,000 informative SNPs. The ranking remains similar with other summary statistics (Fig. 3B and Tables S2-S3 in Additional file 1), except that FImpute3.0 presented the lowest minimum and maximum individual SEC. With FImpute3.0, the lowest value was equal to 2, indicating that almost all chromosomes were perfectly phased for that individual. With ShapeIT4.1 

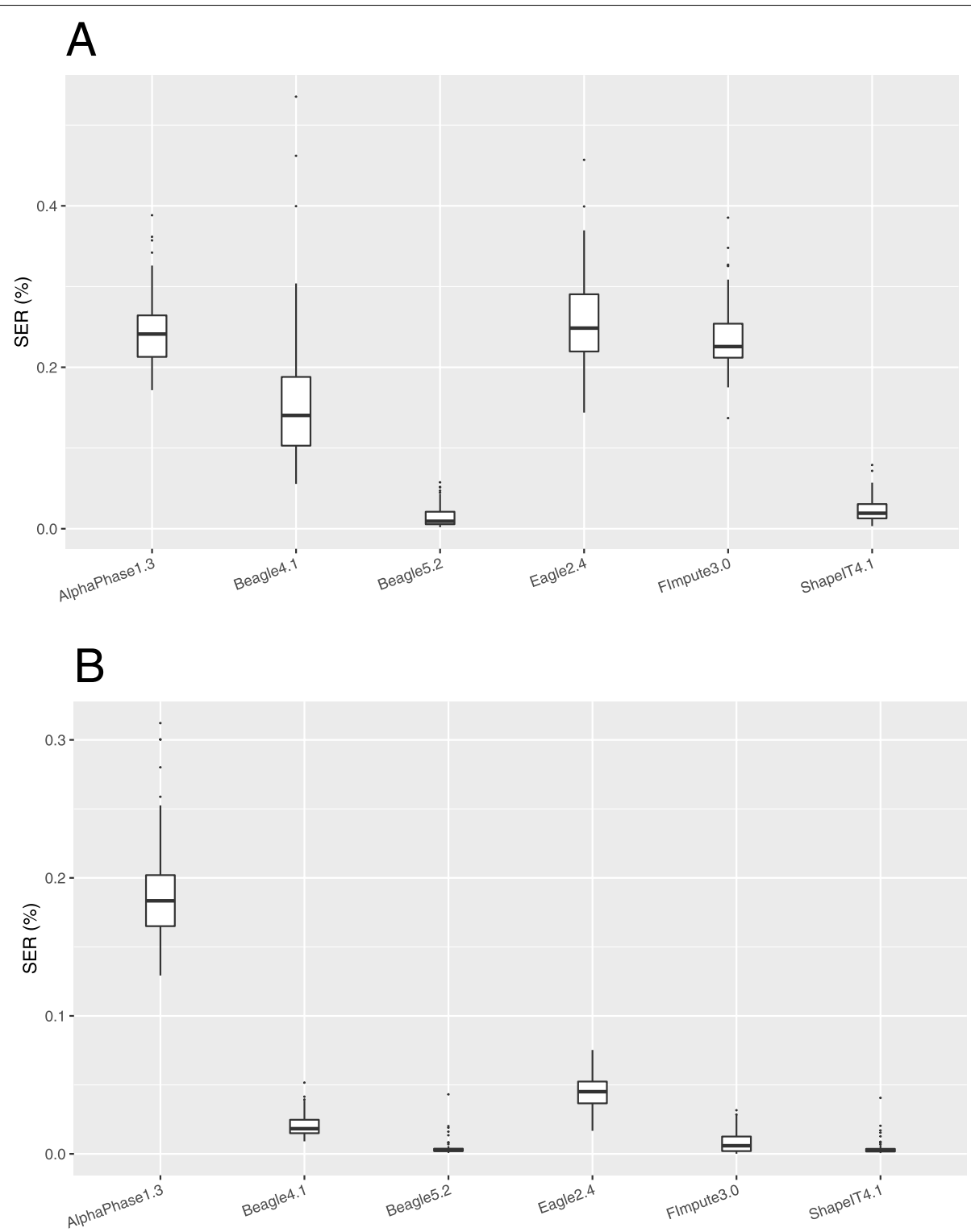

Fig. 3 Switch error rates in both scenarios. Boxplots of switch error rates (SER, \%) obtained with AlphaPhase1.3, Beagle4.1, Beagle5.2, Eagle2.4, FImpute3.0 and ShapelT4.1, computed for the 98 validation individuals (A) in scenario 1 with only the 98 validation individuals, and (B) in scenario 2 with the 264 sequenced individuals

and Beagle5.2, the best phased individual had only 12 and 13 SEC, respectively. The median SEC dropped for all the different versions of the tested software, and the variation among individuals was strongly reduced, in particular for Beagle3.3, Beagle4.0, Beagle4.1 and Beagle 5.1 (Figure S1B in Additional file 1). The ranking of these different versions, in terms of SEC or SER, was similar to the ranking observed in the first scenario, with the exception of ShapeIT2 that presented now higher SEC and variation levels than ShapeIT4.1. Differences between Beagle5.1 and Beagle5.2 were also smaller than in the first scenario.

\section{Length of correctly phased haplotype blocks}

Statistics relying on SEC does not provide a full description of their distribution along the chromosomes and of the resulting distribution of length of correctly phased haplotype segments. Therefore, we also computed the quality adjusted (QA) haplotype block length and the QAN50 metrics, as described in the Methods section, in order to highlight the ability of a phasing tool to produce long correctly phased blocks within a chromosome, without switch error. 
Table 1 Results of different metrics used to assess phasing quality in both scenarios. Median values of switch error counts (SEC), switch error rates (SER, \%), quality adjusted (QA) haplotype block length (bp), and QAN50 (bp), obtained with AlphaPhase1.3, Beagle4.1, Beagle5.2, Eagle2.4, Flmpute3.0 and ShapelT4.1, computed for the 98 validation individuals in each scenario (scenario 1: using the 98 validation individuals; scenario 2: using the 264 sequenced individuals)

\begin{tabular}{|c|c|c|c|c|c|c|c|c|}
\hline Metric & $\begin{array}{l}\text { Median } \\
\text { SEC }\end{array}$ & & $\begin{array}{l}\text { Median } \\
\text { SER (\%) }\end{array}$ & & $\begin{array}{l}\text { Media } \\
\text { haplot }\end{array}$ & ck length (bp) & QAN50 (bp) & \\
\hline Scenario & 1 & 2 & 1 & 2 & 1 & 2 & 1 & 2 \\
\hline AlphaPhase1.3 & 4612 & 3548 & 0.2412 & 0.1834 & 10,653 & 55,973 & $2,505,672$ & $2,719,430$ \\
\hline Beagle4.1 & 2769 & 359 & 0.1404 & 0.0183 & 484 & 45,729 & $18,212,951$ & $44,254,507$ \\
\hline Beagle5.2 & 191 & 55 & 0.0093 & 0.0027 & 12,334 & $6,953,676$ & $47,895,001$ & $62,687,474$ \\
\hline Eagle2.4 & 4901 & 882 & 0.2485 & 0.0451 & 172 & 161 & $5,771,337$ & $22,281,097$ \\
\hline Flmpute3.0 & 4477 & 115 & 0.2256 & 0.0059 & 583 & 27,490 & $6,114,640$ & $79,974,276$ \\
\hline ShapelT4.1 & 386 & 50 & 0.0192 & 0.0026 & 2134 & $4,738,542$ & $48,437,671$ & $69,002,979$ \\
\hline
\end{tabular}

In the first scenario, median QA haplotype block lengths are equal respectively to 10 and $12 \mathrm{~kb}$ with AlphaPhase1.3 and Beagle5.2, and clearly lower with other methods (Table 1). The ranking of the methods based on the median QA haplotype block lengths is thus very different from comparisons based on SEC, with AlphaPhase1.3 ranking second. However, when mean values are used in comparisons (Table S4 in Additional file 1), the ranking follows results obtained with SEC. These mean lengths of correctly phased segments range from $500 \mathrm{~kb}$ with AlphaPhase1.3 to $7.5 \mathrm{Mb}$ with Beagle 5.2. Compared to AlphaPhase1.3, the median values were five times lower with ShapeIT4.1 but the haplotype block lengths were on average ten times longer. This indicates that some methods such as ShapeIT4.1 tend to produce a lot of small correctly phased segments (switch errors being close) in combination with very long correctly phased segments (up to $156.8 \mathrm{Mb}$ with ShapeIT4.1, a full chromosome), whereas other methods such as AlphaPhase 1.3 tend to provide more uniform distances between successive switch errors. This is confirmed in the distributions of QA haplotype block lengths (Figure S3 in Additional file 1) that are concentrated around short values with all methods, although long correctly phased segments are observed, in particular with ShapeIT4.1 and Beagle5.2. As a result, the mean length is higher with these two methods. Long segments capture large fractions of the genome and the QAN metrics provide complementary information by weighting the segments by their length (in $\mathrm{Mb}$ or in number of SNPs). For instance, the QAN50 metrics obtained for different methods (Fig. 4A and Table 1) indicate that with AlphaPhase1.3, $50 \%$ of the genome is included in correctly phased segments longer than $2.5 \mathrm{Mb}$. The QAN50 increases to 5.8 and $6.1 \mathrm{Mb}$ with FImpute3.0 and Eagle2.4, respectively (approximately 20,000 SNPs, Fig. 4C) and to $18.2 \mathrm{Mb}$ with Beagle4.1(approximately 60,000 SNPs). ShapeIT4.1 and Beagle5.2 performed best with a QAN50 close to $48 \mathrm{Mb}$ corresponding to blocks of approximately 170,000 SNPs. Figure 4A provides the full distribution of QAN values (from 100 to $0 \%$ ), with very similar curves for ShapeIT4.1 and Beagle5.2. It allows also to determine the percentage of the genome included in correctly phased segments longer than different thresholds as reported in Table 2. For instance, for applications such as imputation or haplotype-based association studies, phasing accuracy is important locally, at short range $(<1 \mathrm{Mb})$. The aptitude to produce long correctly phased segments $(>10 \mathrm{Mb})$ for most of the genomic positions is more important in applications relative to the age of young alleles, of recent IBD segments or recent selective sweeps. With Beagle5.2 for instance, 96.3, 93.6, 89.3 and $48.1 \%$ of the genome is included in correctly phased segments of at least 1,5 , 10 and $50 \mathrm{Mb}$, respectively. These values are close with ShapeIT4.1 and lower with the remaining methods, only 86.4, 54.0, 33.2 and 1.3\% with Eagle2.4, for instance. Most recent versions of tested software performed better in terms of QA and QAN50 than older versions (Figure S2A, C and Table S4 in Additional file 1), with the exception of ShapeIT2 that had similar statistics as ShapeIT4.1, and Beagle4.1 that presented better results than Beagle5.0.

In the second scenario including more individuals, mean QA haplotype block lengths (Table S4 in Additional file 1) increased for all methods, reaching 23.3 and $24.1 \mathrm{Mb}$ with Beagle5.2 and ShapeIT4.1, respectively. The distributions of QA haplotype block lengths are clearly shifted towards longer segments with ShapeIT4.1 and Beagle5.2, and with FImpute3.0 to a lesser extent (Figure S3 in Additional file 1). Interestingly, the improvement is only modest with AlphaPhase 1.3 whereas the mean QA haplotype block length increases from $500 \mathrm{~kb}$ to $12.5 \mathrm{Mb}$ with FImpute3.0, a 25-fold change. FImpute3.0 is even the best method with 


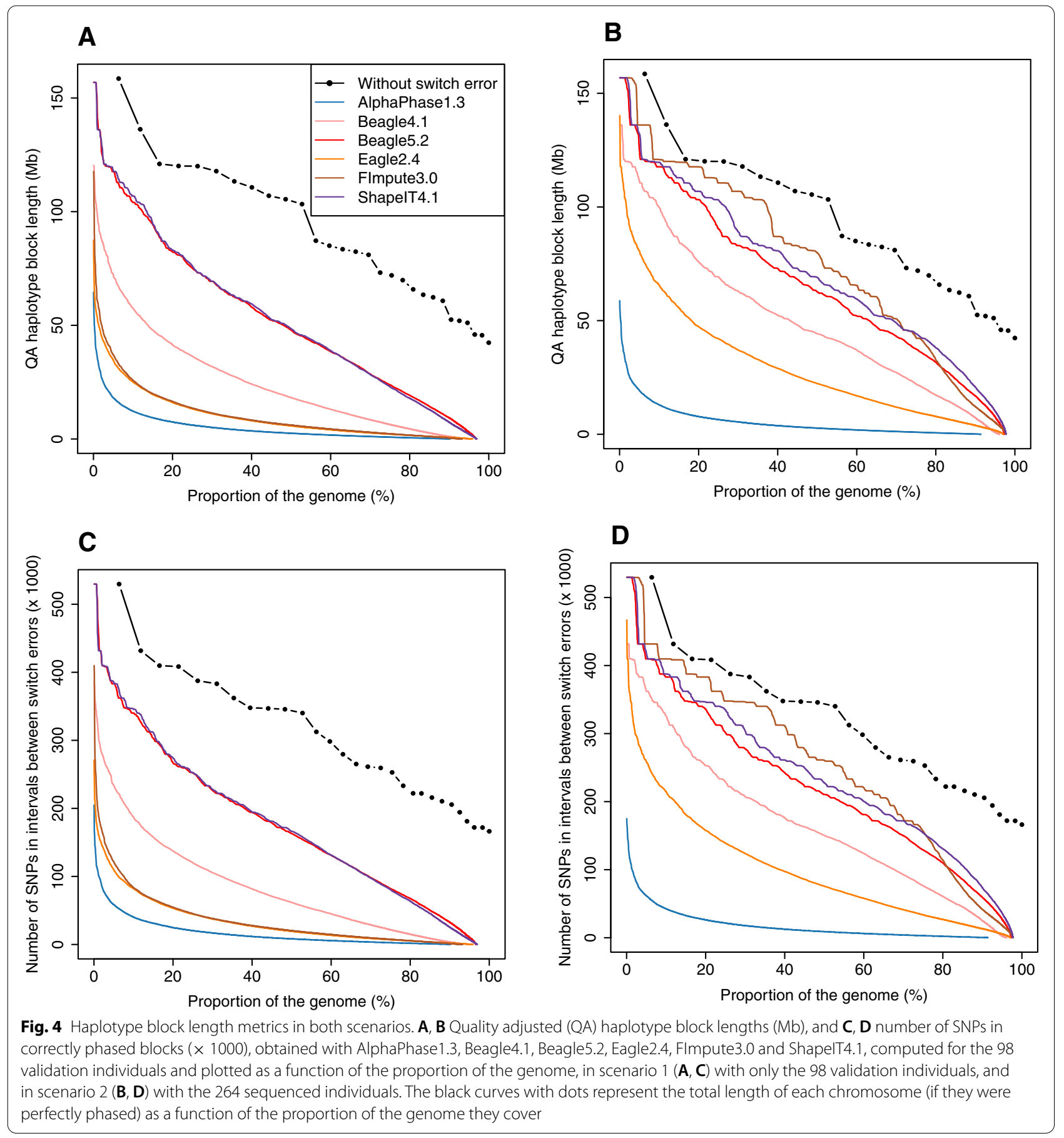

respect to QAN50 (79.9 Mb), followed by ShapeIT4.1 $(69.0 \mathrm{Mb})$ and Beagle5.2 (62.7 Mb) (Table 1). However, a larger proportion of the genome is included in correctly phased segments longer that $10 \mathrm{Mb}$ with ShapeIT4.1 (95.1\%) compared to FImpute3.0 (91.6\%) (Fig. 4B, D and Table 2). ShapeIT4.1 performed slightly better than Beagle5.2 at different thresholds (see Fig. 4B, D). Comparisons of different versions of tested software is in agreement with comparisons made with the first scenario, the differences between Beagle5.0, Beagle5.1 and Beagle 5.2 being however smaller (Figure S2B, D and Table S4 in Additional file 1). 
Table 2 Genome percentage included in correctly phased segments longer than different thresholds in both scenarios. Percentage of the genome covering quality adjusted (QA) haplotype blocks of minimal length of respectively 1, 5, 10 and $50 \mathrm{Mb}$, obtained with AlphaPhase1.3, Beagle4.1, Beagle5.2, Eagle2.4, Flmpute3.0 and ShapelT4.1, computed for the 98 validation individuals in each scenario (scenario 1: using the 98 validation individuals; scenario 2: using the 264 sequenced individuals)

\begin{tabular}{llllllll}
\hline $\begin{array}{l}\text { QA haplotype block } \\
\text { length (Mb) }\end{array}$ & $\mathbf{1}$ & & $\mathbf{5}$ & & $\mathbf{1 0}$ & & $\mathbf{5 0}$ \\
\hline Scenario & 1 & 2 & 1 & 2 & 1 & 2 & 1 \\
AlphaPhase1.3 & 71.5 & 73.7 & 30.4 & 32.2 & 13.6 & 14.5 & 0.2 \\
Beagle4.1 & 90.4 & 94.9 & 78.5 & 91.8 & 66.5 & 87.7 & 13.8 \\
Beagle5.2 & 96.3 & 97.2 & 93.6 & 96.1 & 89.3 & 93.9 & 48.1 \\
Eagle2.4 & 86.4 & 96.2 & 54.0 & 87.2 & 33.2 & 74.4 & 1.3 \\
Flmpute3.0 & 85.6 & 97.5 & 56.1 & 95.2 & 34.0 & 91.6 & 2.1 \\
ShapelT4.1 & 96.1 & 97.6 & 92.2 & 96.8 & 87.6 & 95.1 & 70.1 \\
\hline
\end{tabular}

\section{Pairwise SNP phasing accuracy}

Finally, we compared the methods in terms of pairwise SNP phasing accuracy. This metric represents the probability that there is no phasing error between two SNPs as a function of their distance. The results are reported in Fig. 5 and Table 3 and are in agreement with observations for other metrics such as QAN50. In the first scenario, these probabilities are above 0.95 and 0.92 at 10 and $100 \mathrm{~kb}$, respectively, with Beagle4.1, Beagle5.2 and ShapeIT4.1 (Fig. 5A). Other methods presented values below 0.93 and 0.91 , respectively. The probabilities dropped rapidly at longer distances, even at $1 \mathrm{Mb}$ (around 0.80 with Beagle4.1 and even below 0.70 for the three less efficient methods). ShapeIT4.1 and Beagle5.2 performed best with probabilities still above 0.90 at $1 \mathrm{Mb}$, but only 0.78 and 0.66 at 5 and $10 \mathrm{Mb}$, respectively. At $50 \mathrm{Mb}$, the probabilities were almost null with all methods and only 0.16 and 0.17 with Beagle 5.2 and ShapeIT4.1, respectively. In the second scenario, the probabilities are higher and drop less rapidly, presenting a plateau until a distance of almost $1 \mathrm{Mb}$ (Fig. 5B). ShapeIT4.1 achieved the highest probabilities, equal to $0.95,0.87$ and 0.77 at 1,5 and $10 \mathrm{Mb}$ distance, respectively, but FImpute3.0 achieved almost identical results and was even better at very long distance $(0.31$ at $50 \mathrm{Mb}$ vs 0.27 for ShapeIT4.1).

\section{Discussion}

We herein compared accuracy of population-based phasing tools in a whole-genome sequenced cattle pedigree. To be able to measure the accuracy, it was essential to apply certain procedures to our whole-genome sequencing data. Indeed, after filtering variants based on a standard variant quality score recalibration procedure, the number of $\mathrm{CO}$ in our pedigree was still highly inflated, suggesting that these pedigree-based haplotypes contain many errors. We had to apply further filters to our data to remove additional low-quality markers or small genomic regions incorrectly mapped in the reference genome build. Refining genotype calling with Beagle4.1 [39] had a major impact, stressing the importance of such a procedure. Our final data presented still a few more CO than those obtained at lower density with a high-confidence map. This could be due to the $\mathrm{CO}$ missed at lower density or to the errors that remain in the sequence data. This would represent a maximum of 5 incorrect $\mathrm{CO}$ on average per meiosis, and these errors could result from a phasing error in the parent or in the offspring haplotypes (each of these haplotypes would thus have less than 5 errors). We could apply additional filters to further reduce the number of errors. For instance, we could remove SNPs located in copy number variants since they would generate spurious $\mathrm{CO}$ as genotype calling is more difficult at these positions [41]. Similarly, heterozygous genotypes in the middle of long homozygous-by-descent segments [42] are also probably errors and would generate incorrect $\mathrm{CO}$. Nevertheless, our results illustrate that many errors are still present in whole-genome sequencing data, and that stringent filtering is required. The presence of these low-quality variants would not be a problem in genome-wide association studies because associations are tested independently for each SNP, and genomic prediction methods might be robust to this problem. It might even be key to keep as much variants as possible to have the causative variants in the data set. However, for applications relying on haplotypes and their length, stringent filtering is essential, in particular when long correctly phased segments are required. To illustrate the impact of filtering, we evaluated the methods before improving genotype quality with Beagle 4.1 and observed that the phasing accuracy was strongly reduced (Table S5 and Table S6 in Additional file 1). With ShapeIT4.1, the SER were for instance 6 and 40 times higher, in the first and second scenarios respectively, compared to values 


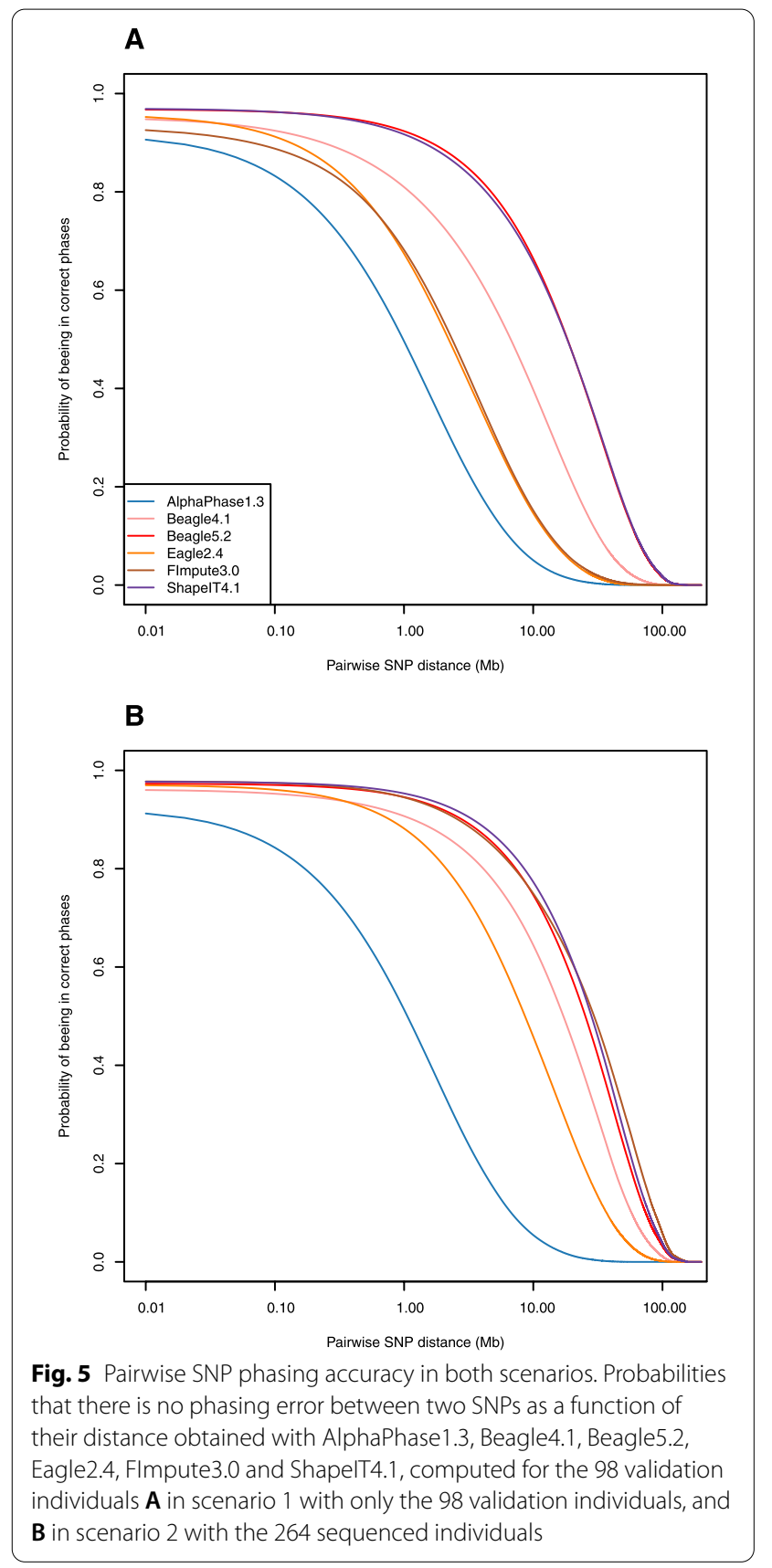

obtained on a cleaned data set with the same software. Similarly, the QAN50 was divided approximately by ten in both scenarios when ShapeIT4.1 was applied to lower quality genotypes. Overall, evaluations on these data resulted in smaller differences between methods and scenarios, indicating that most of the phasing errors result from the presence of genotyping or map errors in the data rather than from differences between phasing approaches. Nevertheless, ShapeIT4.1 and Beagle5.2 remained overall the best, whereas performances from
FImpute3.0 were heavily impacted. This evaluation further stresses the importance to improve as much as possible the genotype quality.

The quality of our final data set was high enough to evaluate the phasing methods, since we expect only a few errors (from 0 to 5 ) in our reference haplotypes. In our best scenario, the accuracy of the best method was impressive with a median of 50 SEC per individual, corresponding to approximately only two SEC per chromosome and to a SER of $0.003 \%$. In the case of point switches, a phasing error at a single marker (or a small segment) that would cause two consecutive SEC (see example in Fig. 2) would represent only one such punctual error per chromosome. Some individuals presented only 2 SEC for their entire genome, and chromosomes were frequently phased without errors. These results are also confirmed with the metrics related to length of correctly phased segments. On average, ShapeIT4.1 had only one switch error every 40,000 informative markers. When only one hundred individuals were simultaneously phased, there were around 10 switches on average per chromosome with Beagle5.2 (one switch error every 10,000 SNPs corresponding to a SER of $0.01 \%$ ). These are nevertheless excellent results given the small sample size. These are indeed better results than those reported in human's populations. For instance, Delaneau et al. [35] obtained a SER above $0.5 \%$ with ShapeIT4 and Beagle 5 and with a reference panel of 20,000 individuals (at lower marker density). Loh et al. [33] obtained also higher SER using ShapeIT2 and Eagle2 whereas Choi et al. [23] estimated that the SER ranged from 0.8 to $1.5 \%$ for Eagle2, ShapeIT2 and Beagle4 for a reference 'Genome-In-ABottle' whole-genome phased individual, and using a reference panel of 2500 individuals. Similarly, Song et al. [13] reported higher SER, above 2\%, in human populations phased with ShapeIT2. This higher accuracy in our cattle data set might be related to the lower effective population size (around 100 in the current population $[43,44]$ ), the higher relatedness (see for instance Figure S4 in Additional file 1) and LD levels, particularly at long distance $(>0.1$ when marker distance $>1 \mathrm{Mb}[45])$. We previously observed that population-based methods such as Beagle are very effective at indirectly exploiting the familial information through the presence of long-shared haplotypes (see also [24]). Similarly, methods such as AlphaPhase [29] or FImpute [30] can identify parents or surrogate parents without pedigree information. This was confirmed in the present study as increasing the sample size and including sequenced relatives clearly improved the accuracy, in particular for FImpute3.0, although the pedigree information was not explicitly used. The high observed accuracy might also result from the stringent rules applied to improve the quality of our data set. With 
Table 3 Pairwise SNP phasing accuracy at different distances in both scenarios. Pairwise SNP phasing accuracy at distances of respectively 0.01, 0.1, 1, 2, 5, 10 and $50 \mathrm{Mb}$, obtained with AlphaPhase1.3, Beagle4.1, Beagle5.2, Eagle2.4, FImpute3.0 and ShapelT4.1, computed for the 98 validation individuals in each scenario (scenario 1: using the 98 validation individuals; scenario 2: using the 264 sequenced individuals)

\begin{tabular}{|c|c|c|c|c|c|c|c|c|c|c|c|c|c|c|}
\hline $\begin{array}{l}\text { Pairwise SNP } \\
\text { distance (Mb) }\end{array}$ & 0.01 & & 0.10 & & 1 & & 2 & & 5 & & 10 & & 50 & \\
\hline Scenario & 1 & 2 & 1 & 2 & 1 & 2 & 1 & 2 & 1 & 2 & 1 & 2 & 1 & 2 \\
\hline AlphaPhase1.3 & 0.91 & 0.91 & 0.83 & 0.84 & 0.50 & 0.51 & 0.33 & 0.35 & 0.14 & 0.15 & 0.05 & 0.05 & 0.00 & 0.00 \\
\hline Beagle4.1 & 0.95 & 0.96 & 0.92 & 0.95 & 0.81 & 0.91 & 0.73 & 0.87 & 0.57 & 0.77 & 0.40 & 0.64 & 0.03 & 0.14 \\
\hline Beagle5.2 & 0.97 & 0.97 & 0.96 & 0.97 & 0.92 & 0.95 & 0.89 & 0.92 & 0.79 & 0.85 & 0.66 & 0.75 & 0.16 & 0.24 \\
\hline Eagle2.4 & 0.95 & 0.97 & 0.91 & 0.96 & 0.67 & 0.88 & 0.52 & 0.81 & 0.30 & 0.64 & 0.15 & 0.46 & 0.00 & 0.05 \\
\hline FImpute3.0 & 0.93 & 0.98 & 0.89 & 0.97 & 0.68 & 0.95 & 0.54 & 0.92 & 0.31 & 0.84 & 0.15 & 0.75 & 0.00 & 0.31 \\
\hline ShapelT4.1 & 0.97 & 0.98 & 0.96 & 0.97 & 0.92 & 0.95 & 0.88 & 0.93 & 0.78 & 0.87 & 0.66 & 0.77 & 0.17 & 0.27 \\
\hline
\end{tabular}

less stringent rules, phasing accuracy dropped indeed significantly (see above).

We herein evaluated the phasing methods with default settings. However, performances from most phasing methods could be further improved by optimizing their parameters. Settings from methods that were originally developed for human populations might indeed not be optimal for livestock populations. For instance, we observed that accuracy of ShapeIT4.1 could be slightly improved by increasing the value of the --pbwt-depth parameter in the second scenario (Table S7 and Table S8 in Additional file 1). This parameter defines the number of selected conditioning neighboring haplotypes to perform a Li and Stephens model [40], higher values increase the accuracy but also the computational costs [35]. However, the optimal parameters for each method might depend on the population structure, the number of individuals, the marker density, etc. Therefore, it is difficult to select optimal values prior to the analysis, and we preferred to compare the phasing methods with their default settings.

In our study, ShapeIT4.1 and Beagle5.2 performed best for almost all evaluated metrics and for both scenarios. Their relative ranking varied however according to the metric and the scenario. Beagle5.2 achieved the best results mainly in the first scenario whereas ShapeIT4.1 was often the most accurate in the second scenario. When the parents were included in the data, FImpute3.0 accurately phased extremely long segments and the estimated SEC was as low as 2 for some individuals. Nevertheless, when the parents were not included in the sample, the accuracy of FImpute3.0 decreased although some full-sibs were present in the sample (Figure S4 in Additional file 1). Phasing accuracy varies across different versions of a software. In our study, we observed that phasing accuracy improved as expected with newer versions of the software. As a result, comparisons of different methods might vary through time, according to the compared versions. For instance, until recently ShapeIT4.1 was in competition with Beagle5.1. In our comparisons, ShapeIT4.1 was most often better although Beagle5.1 performed extremely well. However, Beagle5.2, the new release, performed as well as ShapeIT4.1 (see above). Phasing accuracy will also change according to different elements such as marker density, level of relatedness and size of the population [24], and this might impact the ranking of the methods. For instance, in Choi et al. [23], Eagle2 performed better than ShapeIT2 and Beagle4 on human data. In a Holstein dairy cattle population genotyped with medium to high density genotyping arrays, Miar et al. [46] compared Beagle4.1, ShapeIT2 and FImpute based on SER. They estimated that Beagle4.1 was the most accurate whereas ShapeIT2 resulted in higher SER. However, their sample was much larger and more information from relatives was thus available. Consistently with our study, when one or two parents of the validation animals were added to the phased sample, FImpute became more accurate than Beagle4.1. Using simulated data mimicking a brown layer population, Frioni et al. [47] observed that haplotypes phased with Beagle4.1 had lower SEC than those obtained with FImpute when parents were not included. As in our study, inclusion of parents in the phased sample increased phasing accuracy for FImpute. Fewer comparisons in livestock species are available for ShapeIT4.1 or Beagle5.0 as these programs are more recent. In summary, our data set represent a typical example of reference panel containing 100 to 200 whole-genome sequenced individuals in a livestock species with high levels of relatedness. In those conditions, ShapeIT4.1 and Beagle5.2 performed particularly well. We are not aware of the reasons why these two approaches present higher phasing accuracies. Both of them rely on a $\mathrm{Li}$ and Stephens model [40] that might result more flexible and more accurate than 
methods relying on matching haplotypes of fixed length such as AlphaPhase or FImpute. Browning and Browning [24] previously reported that the original phasing algorithm from Beagle (implemented in Beagle3 or Beagle4) was less efficient with smaller samples (as in the present study). The implementation of the method, including parameters setting and fine-tuning, impacts also the phasing accuracy as we observed that methods with the same global approach (e.g., the Li and Stephens model), or even successive versions of the same software, achieve different accuracies. Similarly, it is difficult to determine the cause of the poorer performances of AlphaPhase, although lower phasing accuracy compared to Eagle2 was already reported in the study presenting the software [48].

The choice of the phasing method might nevertheless depend on the availability of other options. For instance, Beagle4.0, FImpute3.0 and AlphaPhase1.3 can exploit the pedigree information, which might increase their phasing accuracy in certain conditions. When pedigree information was used in the first scenario (without the sequenced parents), phasing accuracy of AlphaPhase or FImpute was however not higher (Table S9 and Table S10 in Additional file 1) probably because that information was already captured through long haplotype sharing between individuals or because these approaches can identify parents or surrogate parents without the need of the pedigree information. The benefit of pedigree information is stronger when direct relatives such as sequenced parent or offspring are available as in the second scenario, or at lower marker density when LD methods are less efficient. Familial information can also be integrated in some LD-based approaches with a two-step procedure in which haplotypes are first obtained based on familial information and unphased markers are subsequently phased by a LD-based approach [26]. Such an approach is possible with Beagle4.1 or ShapeIT4.1, that will preserve pre-phasing information present in the VCF file. Phasing information coming from marker alleles present on the same sequenced reads can also be integrated with such an approach.

Finally, the importance of phasing accuracy will depend on the applications in which the haplotypes are used. For many applications, accurate phasing is only required at short range. For haplotype-based association studies, short 100-kb haplotypes would capture interactions among tightly-linked loci. We previously observed that improved long-range phasing accuracy did not result in higher imputation accuracy in a livestock population [49]. The presence of a few switch errors would not necessarily be a problem in haplotype-based GWAS or genomic selection, or in some QTL mapping approaches, as long as correctly phased segments are long enough to infer the IBD relationships around the tested position. For such applications, most of the tested methods would provide sufficient accuracy. The phasing accuracy will be more important in applications in which the length of shared haplotypes is used to estimate age of alleles [17] or age to a common ancestor, to identify signatures of selection $[15,16]$, to determine relatedness between individuals based on the distribution of length of shared IBD segments [4]. This accuracy will also be essential in studies on meiotic recombination based on the identification of $\mathrm{CO}$ in genotyped or sequenced pedigrees $[20,50]$.

\section{Methods}

\section{Sequencing data}

The whole-genome sequence data used in the present work was obtained from 264 Holstein-Friesian individuals from the DAMONA pedigree designed to study germline mutation in cattle [51] and previously used and described $[52,53]$. The individuals were sequenced at high coverage (mean coverage: $25.8 \mathrm{X}$, ranging from 15.2X to $47.1 \mathrm{X}$ ), and the data included 98 sequenced trios (Figure S3 in Additional file 1). Whole genome Illumina Nextera PCR free libraries (550 bp insert size) were sequenced on an Illumina HiSeq 2000 with a paired-end protocol $(2 \times 100 \mathrm{bp})$.

The sequencing data was re-aligned on the new ARSUCD1.2 (BosTau9) bovine genome assembly [37] using the Burrows-Wheeler Aligner MEM algorithm (v0.7.5a) [54]. The SAM files were converted into BAM files with SAMtools (v1.9) [55]. The BAM files were sorted using Sambamba (v0.6.6) [56]. PCR duplicates were marked with the MarkDuplicates option of picard-tools (v2.7.1) [57]. The BAM files were then recalibrated using the BaseRecalibrator procedure of GATK (v4.1.7.0) [58-60], using the VCF provided by the 1000 Bull Genome project (http://www.1000bullgenomes.com/) as known polymorphic sites database. Individual GVCF files were obtained with HaplotypeCaller (GATK4) and were subsequently merged in a GenomicsDB (with GenomicsDBImport, GATK4) to perform joint genotyping with GenotypeGVCFs (GATK4). Variants from the resulting VCF file were then recalibrated using VariantRecalibrator (GATK4) by applying two thresholds (99.9 and 97.5) and using $1.2 \mathrm{M}$ SNPs extracted from commercial chips [61] as truth and training sets, and $138 \mathrm{M}$ SNPs provided by the 1000 Bull Genome project as known set.

\section{Assessing the quality of whole-genome sequence genotype data Strategy}

To evaluate the quality of whole-genome sequence data in terms of genotyping error rates and of physical marker order, we compared the number of $\mathrm{CO}$ identified in our 
pedigree with the sequence data to the number of $\mathrm{CO}$ identified in our pedigree with a lower density highconfidence map validated on a much larger population genotyped with the Illumina BovineSNP50 BeadChip (Illumina Inc., San Diego, CA). The CO are identified in sequenced parent-offspring pairs, also referred to as proband and gamete. For the comparisons, we relied only on the most accurate $\mathrm{CO}$ counts, obtained when at least one parent of the proband was also sequenced (160 gametes out of 279: 104 females and 56 males probands). Although a few CO might be missed or incorrectly identified with this reference map, much higher counts of $\mathrm{CO}$ with the sequence data would indicate presence of genotyping and map errors.

\section{Validation of the reference $50 \mathrm{~K}$ map}

To obtain a high-confidence reference $50 \mathrm{~K}$ marker map, we used the genotype data from Kadri et al. [52], available for 115,967 individuals and 30,349 SNPs and followed their approach relying on the map confidence score (MCS) implemented in LINKPHASE3 [36] to identify eventually misplaced markers that were subsequently removed. Physical positions of the markers in the ARSUCD1.2 bovine genome assembly were obtained from https://www.animalgenome.org/repository/cattle/UMC bovine_coordinates. Among the 30,331 SNPs from this high-confidence reference map, 29,375 SNPs were present in our whole-genome sequence data. The resulting high-confidence reference $50 \mathrm{~K}$ marker map is provided in Additional file 2.

\section{Evaluated data processing steps}

Variant quality score recalibration (VQSR) By applying the VariantRecalibrator procedure (GATK, see above) on the genotype calls, we kept 15,327,429 and 11,030,905 SNPs with the 99.9 and 97.5 thresholds, respectively. These variants correspond to 99.9 and $97.5 \%$ of the total truth sites, 95.1 and $76.4 \%$ of the total known SNPs, and 60.1 and $16.0 \%$ of the total novel SNPs, respectively.

Variant selection based on genetic rules To further enrich our data set in high-quality variants, we selected variants behaving as true SNPs based on Hardy-Weinberg equilibrium test $(p>0.05)$, with expected genotype frequencies in offspring from heterozygous parents (based on a $\chi^{2}$ test, $p>0.05$ ), and not presenting more than one Mendelian inconsistency in parent-offspring pairs or trios (e.g., opposite homozygotes) for the entire pedigree. We kept only variants for which the probability to observe no Mendelian inconsistencies by chance was lower than 1e-12. Finally, we also discarded uninformative markers with low minor allele frequency $(<0.01)$.
Applications of these rules resulted in the selection of $8,435,899$ informative variants behaving as true SNPs.

Improving genotyping-calling The accuracy of genotyping-calling for these 8,435,899 variants was then improved with the LD-based approach implemented in Beagle4.1 [39].

Exclusion of suspicious genomic regions Several filters were applied to exclude genomic regions of putatively lower quality. First, we removed the last 10-kb segments at chromosome ends. Second, we excluded high coverage regions that we identified within each individual using the approach described in the LUMPY framework [62]. We considered as high coverage regions the genomic regions presenting 6 times higher coverage than the individual average whole-genome coverage. Finally, we discarded genomic regions associated with putative errors in the genome assembly. To that end we used tools available in LINKPHASE3 [36] and an approach described in more details in Kadri et al. [41]. We first identified small genomic regions flanked by peaks of high recombination rate $(>0.05)$. For these genomic regions, we compared within-family segregation patterns (inheritance vectors as described in Druet and Georges [36]) to those obtained with the high-confidence $50 \mathrm{~K}$ map in the flanking regions, and removed those with a squared correlation $<0.90$. We subsequently removed SNPs with a low MCS $(<0.99)$ as well as chromosome extremities when they presented inflated recombination rate with flanking regions. The procedure was repeated until no further evidence for map errors was visible. In total, we excluded 18,220 SNPs associated with these regions (Additional file 3).

Final editions We finally set to missing 38,537 incompatible genotypes in parent-offspring pairs (opposite homozygous), eventually introduced after running Beagle4.1. We also removed SNPs that were monomorphic at this stage and those with a missing genotyping rate above $5 \%$ in the final pedigree. The final number of SNPs was 8,417,679 (complete list provided in Additional file 4).

\section{Phasing methods}

We herein compared the accuracy from different phasing software: AlphaPhase v1.1 [29] and v1.3 [48], ShapeIT v2.r904 [31] and v4.1.3 [35], Beagle v3.3.2, v4.0, v4.1, v5.0, v5.1, and v5.2 [7, 63], Eagle v2.4.1 [33], and FImpute v3.0 [30]. Most of these methods are population-based methods. Indeed, Eagle and ShapeIT are hidden Markov models (HMM) modeling target 
haplotypes as mosaic of reference haplotypes, similarly to the $\mathrm{Li}$ and Stephens model [40]. The earlier versions of Beagle (v3.3.2, v4.0 and v4.1 [7]) condense all the observed haplotypes into a directed acyclic graph and the resulting model is a variable length Markov chain that can also be viewed as a HMM. The newer versions of Beagle (v5.0, v5.1 and v5.2 [63]) are based on the Li and Stephens HMM [40]. AlphaPhase and FImpute are both heuristic methods performing haplotype matching in fixed-length windows, and that exploit also the pedigree information when possible. More detailed descriptions of these models are available in the original papers. For each of the tools, we used the default parameters.

Comparisons were first realized with the most recent version of different software, including AlphaPhase1.3, ShapeIT4.1, Beagle4.1, Beagle5.2, Eagle2.4 and FImpute3.0. We included Beagle4.1 because the method is very different from Beagle5.2. Then, we compared different versions of some software to evaluate the benefit of different updates and to obtain metrics for older versions that have been compared in previous studies, or that are sometimes still in use. This allows also to evaluate whether it is worth re-phasing a data set after the release of a new version. FImpute3.0 and Eagle2.4 were not included in this comparison as we only tested one version for these software.

\section{Evaluation of haplotype phasing quality Gold standard for haplotype evaluation}

In real data sets, true haplotypes remain generally unknown. Therefore, we rely on a family-based strategy that is possible thanks to the availability of a sequenced pedigree $[24,64]$. In that case, child's haplotypes can be resolved using Mendelian segregation rules when at least one of their parents is also sequenced. In the absence of genotyping or marker order errors, this results in exact haplotypes although some positions might remain unphased. To minimize those errors in our data set, we applied the different procedures described above. The family-based haplotypes can then be used to assess the quality of haplotypes obtained using population-based phasing methods. In our sequenced pedigree, we had 98 sequenced offspring with both parents sequenced (trios). We phased these trios using Mendelian segregation rules with LINKPHASE3 [36] and used them subsequently as the gold standard (referred to as "true haplotypes"). In the first tested scenario, the sample of 98 offspring (validation individuals) was phased with each method. In a second scenario, all the 264 sequenced individuals were included in the phasing step, including the sequenced parents but the pedigree information was ignored.
This second scenario allowed to study the impact of increasing the sample size and including more related individuals.

\section{Metrics for phasing performance}

Phasing yield This metric defined as the percentage of phased single nucleotide variants, and representing the completeness of phased haplotypes, is sometimes used in evaluation of phasing quality (e.g., [23]). However, all the evaluated population-based methods achieve $100 \%$ phasing yield and this metric is therefore only relevant for AlphaPhase and FImpute.

Switch error counts and rates For each evaluated phasing method, we compared within individual the combinations of alleles at each pair of consecutive informative sites (i.e. heterozygous and phased in the true haplotype) in the inferred haplotypes with the combination of alleles present in the true haplotypes as illustrated in Fig. 2. Each discrepancy is called a switch error and the total number of switch errors per individual is the switch error count (SEC). Since this number depends on the number of informative SNPs, that represents also the number of opportunities for switch errors, we also defined the switch error rate (SER) as the SEC divided by the number of informative markers (e.g., [23]).

Length of correctly phased haplotype blocks In order to characterize the distance between successive switch errors (distribution along the genome), we used the quality adjusted (QA) haplotype block length and the QAN50 metrics described in Duitama et al. [65]. Briefly, the QA haplotype block length is defined as the length of a segment between two successive switch errors (Fig. 2) multiplied by the proportion of phased SNPs. The QAN50 is defined as the longest QA length such that $50 \%$ of all the informative SNPs are located within haplotype blocks with a QA length larger than QAN50 [4]. In other words, this means that $50 \%$ of the informative SNPs are contained in haplotype blocks of at least QAN50. Note that the quality adjustment was only relevant for methods with a phasing yield below $100 \%$. For other methods, these metrics represent raw block lengths.

Pairwise SNP phasing accuracy Finally, we computed the probabilities that there are no phasing errors between two SNPs as a function of the distance between SNPs pairs, providing a complementary measure to previous metrics and inspired from Choi et al. [23]. 


\section{Abbreviations}

CO: Cross-Over; IBD: Identical-By-Descent; LD: Linkage Disequilibrium; MCS: Map Confidence Score; QA: Quality Adjusted; QTL: Quantitative-Trait Locus; SEC: Switch Error Count; SER: Switch Error Rate; VQSR: Variant Quality Score Recalibration.

\section{Supplementary Information}

The online version contains supplementary material available at https://doi. org/10.1186/s12864-022-08354-6.

Additional file 1: Figure S1. Switch error rates in earlier versions of evaluated software in both scenarios. Boxplots of switch error rates (SER, \%) obtained with AlphaPhase1.1, AlphaPhase1.3, Beagle3.3, Beagle4.0, Beagle4.1, Beagle5.0, Beagle5.1, Beagle5.2, ShapelT2 and ShapelT4.1, computed for the 98 validation individuals (A) in scenario 1 with only the 98 validation individuals, and (B) in scenario 2 with the 264 sequenced individuals. Figure S2. Haplotype block length metrics in earlier versions of evaluated software in both scenarios. (A, B) Quality adjusted (QA) haplotype block lengths (Mb), and (C, D) number of SNPs in correctly phased blocks ( $\times 1000)$, obtained with AlphaPhase 1.1, AlphaPhase 1.3, Beagle3.3, Beagle4.0, Beagle4.1, Beagle5.0, Beagle5.1, Beagle5.2, ShapelT2 and ShapelT4.1, computed for the 98 validation individuals and plotted as a function of the proportion of the genome, in scenario $1(A, C)$ with only the 98 validation individuals, and in scenario 2 (B, D) with the 264 sequenced individuals. The black curves with dots represent the total length of each chromosome (if they were perfectly phased) as a function of the proportion of the genome they cover. Figure S3. Distribution of haplotype block lengths in both scenarios. The quality adjusted (QA) haplotype block lengths (Mb) were obtained with AlphaPhase1.3, Beagle4.1, Beagle5.2, Eagle2.4, Flmpute3.0 and ShapelT4.1, computed for the 98 validation individuals (A) in scenario 1 with only the 98 validation individuals, and (B) in scenario 2 with the 264 sequenced individuals. Red stars represent mean values. Figure S4. Pedigree tree of the 264 Holstein-Friesian cattle used in this study. Individuals in blue are males, the ones in orange are females, and the ones surrounded in red are the 98 validation individuals (offspring from trios, that is with both parent sequenced) used to compare the algorithms. Table S1. Number of SNPs per chromosome in both scenarios. Total number of whole-genome sequence SNPs and mean numbers of informative SNPs for each chromosome in each scenario (scenario 1: using the 98 validation individuals; scenario 2: using the 264 sequenced individuals). Table S2. Summary statistics of the switch error counts in both scenarios. Minimum, mean, median and maximum switch error counts (SEC), obtained with all the evaluated LD-based phasing algorithms, computed for the 98 validation individuals in each scenario (scenario 1: using the 98 validation individuals; scenario 2: using the 264 sequenced individuals). Table S3. Summary statistics of the switch error rates in both scenarios. Minimum, mean, median and maximum switch error rates (SER, $\%)$, obtained with all the evaluated LD-based phasing algorithms, computed for the 98 validation individuals in each scenario (scenario 1: using the 98 validation individuals; scenario 2: using the 264 sequenced individuals). Table S4. Summary statistics of the quality adjusted haplotype block lengths in both scenarios. Minimum, mean, median and maximum quality adjusted (QA) haplotype block lengths, obtained with all the evaluated LD-based phasing algorithms, computed for the 98 validation individuals in each scenario (scenario 1: using the 98 validation individuals; scenario 2: using the 264 sequenced individuals). Table S5. Results of different metrics used to assess phasing quality in both scenarios before improving genotype quality with Beagle4.1. Median values of switch error counts (SEC), switch error rates (SER, \%), quality adjusted (QA) haplotype block length (bp), and QAN50 (bp), obtained with AlphaPhase1.3, Beagle4.1, Beagle5.2, Eagle2.4, FImpute3.0 and ShapelT4.1, computed for the 98 validation individuals in each scenario (scenario 1: using the 98 validation individuals; scenario 2: using the 264 sequenced individuals). Table S6. Genome percentage included in correctly phased segments longer than different thresholds in both scenarios before improving genotype quality with Beagle4.1. Percentage of the genome covering quality adjusted (QA) haplotype blocks of minimal length of respectively 1, 5, 10 and $50 \mathrm{Mb}$, obtained with AlphaPhase1.3, Beagle4.1, Beagle5.2,
Eagle2.4, FImpute3.0 and ShapelT4.1, computed for the 98 validation individuals in each scenario (scenario 1: using the 98 validation individuals; scenario 2: using the 264 sequenced individuals). Table S7. Results of different metrics used to assess phasing quality in the second scenario using different parameter settings with ShapelT4.1. Median values of switch error counts (SEC), switch error rates (SER, \%), quality adjusted (QA) haplotype block length (bp), and QAN50 (bp), obtained with ShapelT4.1 using different values $(4,8,16,32,64$ and 128) of the parameter--pbwtdepth, computed for the 98 validation individuals (scenario 2: using the 264 sequenced individuals). Table $\mathbf{S 8}$. Genome percentage included in correctly phased segments longer than different thresholds in the second scenario using different parameter settings with ShapelT4.1. Percentage of the genome covering quality adjusted (QA) haplotype blocks of minimal length of respectively $1,5,10$ and $50 \mathrm{Mb}$, obtained with ShapelT4.1 using different values $(4,8,16,32,64$ and 128) of the parameter --pbwtdepth, computed for the 98 validation individuals (scenario 2: using the 264 sequenced individuals). Table S9. Results of different metrics used to assess phasing quality in the first scenario without and with the pedigree information. Median values of switch error counts (SEC), switch error rates (SER, \%), quality adjusted (QA) haplotype block length (bp), and QAN50 (bp), obtained with AlphaPhase1.1 and FImpute3.0 without and with (ped) the pedigree information, computed for the 98 validation individuals (scenario 1: using the 98 validation individuals). Table S10. Genome percentage included in correctly phased segments longer than different thresholds in the first scenario without and with the pedigree information. Percentage of the genome covering quality adjusted (QA) haplotype blocks of minimal length of respectively 1,5,10 and $50 \mathrm{Mb}$, obtained with AlphaPhase 1.1 and Flmpute 3.0 without and with (-ped) the pedigree information, computed for the 98 validation individuals (scenario 1: using the 98 validation individuals).

\section{Additional file 2.}

Additional file 3.

Additional file 4.

\section{Acknowledgements}

We thank Erik Mullaart and CRV (Arnhem, The Netherlands) for providing the samples. Tom Druet is Senior Research Associate from the Fonds de la Recherche Scientifique - FNRS (F.R.S.-FNRS). Computational resources have been provided by the Consortium des Équipements de Calcul Intensif (CÉCI), funded by the Fonds de la Recherche Scientifique - FNRS (F.R.S.-FNRS) under Grant No. 2.5020.11 and by the Walloon Region. The authors also acknowledge use of the GIGA high performance computing cluster for conducting the study reported in this paper.

\section{Authors' contributions}

LK, WC, GCMM and MG provided data and resources. CO, NK and TD performed the experiments, analyzed and interpreted the data. CO and TD wrote the manuscript. All authors read and approved the final manuscript.

\section{Funding}

This work was funded by the European Research Council (award number: ERC AdG-GA323030, “DAMONA” project) and the Fonds de la Recherche Scientifique-FNRS (F.R.S.-FNRS) under Grant T.0080.20 ("LoCO motifs" research project).

\section{Availability of data and materials}

All relevant data supporting the conclusions of this article are included in the article and its supplementary files. The datasets analyzed during the current study are not yet publicly available because they will be released after the publication of another study, but are available from the corresponding author on reasonable request.

\section{Declarations}

Ethics approval and consent to participate

Not applicable. 


\section{Consent for publication \\ Not applicable.}

\section{Competing interests}

The authors declare that they have no competing interests.

\section{Author details}

'Unit of Animal Genomics, GIGA-R and Faculty of Veterinary Medicine, University of Liège (B34), 4000 Liège, Belgium. ${ }^{2}$ Animal Genomics, ETH Zürich, 8092, Zürich, Switzerland. ${ }^{3}$ Genomics Platform, GIGA, University of Liège (B34), 4000 Liège, Belgium.

Received: 28 October 2021 Accepted: 24 January 2022

Published online: 15 February 2022

\section{References}

1. Marchini J, Howie B. Genotype imputation for genome-wide association studies. Nat Rev Genet. 2010;11(7):499-511.

2. Howie B, Fuchsberger C, Stephens M, Marchini J, Abecasis GR. Fast and accurate genotype imputation in genome-wide association studies through pre-phasing. Nat Genet. 2012;44(8):955-9.

3. Browning SR. Estimation of pairwise identity by descent from dense genetic marker data in a population sample of haplotypes. Genetics. 2008;178(4):2123-32.

4. Gusev A, Lowe JK, Stoffel M, Daly MJ, Altshuler D, Breslow JL, et al. Whole population, genome-wide mapping of hidden relatedness. Genome Res. 2009;19(2):318-26.

5. Druet T, Farnir FP. Modeling of identity-by-descent processes along a chromosome between haplotypes and their genotyped ancestors. Genetics. 2011;188(2):409-19.

6. Meuwissen THE, Karlsen A, Lien S, Olsaker I, Goddard ME. Fine mapping of a quantitative trait locus for twinning rate using combined linkage and linkage disequilibrium mapping. Genetics. 2002;161(1):373-9.

7. Browning SR, Browning BL. Rapid and accurate haplotype phasing and missing-data inference for whole-genome association studies by use of localized haplotype clustering. Am J Hum Genet. 2007;81(5):1084-97.

8. Su S-Y, Balding DJ, Coin LJM. Disease association tests by inferring ancestral haplotypes using a hidden markov model. Bioinformatics. 2008;24(7):972-8.

9. Zhang Z, Guillaume F, Sartelet A, Charlier C, Georges M, Farnir F, et al. Ancestral haplotype-based association mapping with generalized linear mixed models accounting for stratification. Bioinformatics. 2012;28(19):2467-73.

10. de Roos APW, Schrooten C, Druet T. Genomic breeding value estimation using genetic markers, inferred ancestral haplotypes, and the genomic relationship matrix. J Dairy Sci. 2011;94(9):4708-14.

11. Cuyabano BC, Su G, Lund MS. Genomic prediction of genetic merit using LD-based haplotypes in the Nordic Holstein population. BMC Genomics. 2014;15(1):1171.

12. Hess M, Druet T, Hess A, Garrick D. Fixed-length haplotypes can improve genomic prediction accuracy in an admixed dairy cattle population. Genet Sel Evol. 2017;49(1):54.

13. Song S, Sliwerska E, Emery S, Kidd JM. Modeling human population separation history using physically phased genomes. Genetics. 2017:205(1):385-95

14. Speidel L, Forest M, Shi S, Myers SR. A method for genome-wide genealogy estimation for thousands of samples. Nat Genet. 2019:51(9):1321-9.

15. Sabeti PC, Reich DE, Higgins JM, Levine HZP, Richter DJ, Schaffner SF, et al. Detecting recent positive selection in the human genome from haplotype structure. Nature. 2002;419(6909):832-7.

16. Voight BF, Kudaravalli S, Wen $X$, Pritchard JK. A map of recent positive selection in the human genome. PLoS Biol. 2006;4(3):e72

17. Albers PK, McVean G. Dating genomic variants and shared ancestry in population-scale sequencing data. PLoS Biol. 2020;18(1):e3000586.

18. Chan AH, Jenkins PA, Song YS. Genome-wide fine-scale recombination rate variation in Drosophila melanogaster. PLoS Genet. 2012;8(12):e1003090.
19. Kong A, Thorleifsson G, Stefansson H, Masson G, Helgason A, Gudbjartsson DF, et al. Sequence variants in the RNF212 gene associate with genome-wide recombination rate. Science. 2008;319(5868):1398-401.

20. Chowdhury R, Bois PRJ, Feingold E, Sherman SL, Cheung VG. Genetic analysis of variation in human meiotic recombination. PLoS Genet. 2009;5(9):e1000648.

21. Thompson EA. Identity by descent: variation in meiosis, across genomes, and in populations. Genetics. 2013;194(2):301-26.

22. Tewhey R, Bansal V, Torkamani A, Topol EJ, Schork NJ. The importance of phase information for human genomics. Nat Rev Genet. 2011;12(3):215-23.

23. Choi Y, Chan AP, Kirkness E, Telenti A, Schork NJ. Comparison of phasing strategies for whole human genomes. PLoS Genet. 2018;14(4):e1007308.

24. Browning SR, Browning BL. Haplotype phasing: existing methods and new developments. Nat Rev Genet. 2011;12(10):703-14.

25. Williams AL, Housman DE, Rinard MC, Gifford DK. Rapid haplotype inference for nuclear families. Genome Biol. 2010;11(10):R108.

26. Druet T, Georges M. A hidden Markov model combining linkage and linkage disequilibrium information for haplotype reconstruction and quantitative trait locus fine mapping. Genetics. 2010;184(3):789-98.

27. Scheet P, Stephens M. A fast and flexible statistical model for large-scale population genotype data: applications to inferring missing genotypes and Haplotypic phase. Am J Hum Genet. 2006;78(4):629-44.

28. Delaneau O, Marchini J, Zagury J-F. A linear complexity phasing method for thousands of genomes. Nat Methods. 2012;9(2):179-81.

29. Hickey JM, Kinghorn BP, Tier B, Wilson JF, Dunstan N, van der Werf JH. A combined long-range phasing and long haplotype imputation method to impute phase for SNP genotypes. Genet Sel Evol. 2011;43(1):12.

30. Sargolzaei M, Chesnais JP, Schenkel FS. A new approach for efficient genotype imputation using information from relatives. BMC Genomics. 2014;15(1):478

31. O'Connell J, Gurdasani D, Delaneau O, Pirastu N, Ulivi S, Cocca M, et al. A general approach for haplotype phasing across the full spectrum of relatedness. PLoS Genet. 2014;10(4):e1004234.

32. Kong A, Masson G, Frigge ML, Gylfason A, Zusmanovich P, Thorleifsson G, et al. Detection of sharing by descent, long-range phasing and haplotype imputation. Nat Genet. 2008;40(9):1068-75.

33. Loh P-R, Danecek P, Palamara PF, Fuchsberger C, Reshef YA, Finucane KH, et al. Reference-based phasing using the haplotype reference consortium panel. Nat Genet. 2016;48(11):1443-8.

34. Browning BL, Zhou Y, Browning SR. A one-penny imputed genome from next-generation reference panels. Am J Hum Genet. 2018;103(3):338-48.

35. Delaneau O, Zagury J-F, Robinson MR, Marchini JL, Dermitzakis ET. Accurate, scalable and integrative haplotype estimation. Nat Commun. 2019;10(1):5436.

36. Druet T, Georges M. LINKPHASE3: an improved pedigree-based phasing algorithm robust to genotyping and map errors. Bioinformatics. 2015;31(10):1677-9.

37. Rosen BD, Bickhart DM, Schnabel RD, Koren S, Elsik CG, Tseng E, et al. De novo assembly of the cattle reference genome with single-molecule sequencing. GigaScience. 2020;9(giaa021):1-9. https://doi.org/10.1093/ gigascience/giaa021.

38. Qanbari S, Wittenburg D. Male recombination map of the autosomal genome in German Holstein. Genet Sel Evol. 2020;52(1):73.

39. Browning BL, Yu Z. Simultaneous genotype calling and haplotype phasing improves genotype accuracy and reduces false-positive associations for genome-wide association studies. Am J Hum Genet. 2009;85(6):847-61.

40. Li N, Stephens M. Modeling linkage disequilibrium and identifying recombination hotspots using single-nucleotide polymorphism data. Genetics. 2003;165(4):2213-33.

41. Kadri NK, Harland C, Charlier C, Karim L, Cambisano N, Deckers M, et al. High resolution mapping of cross-over events in cattle using NGS data. In: Proceedings of the world congress on genetics applied to livestock production. Auckland; 2018. p. 7. https://www.wcgalp.org/system/files/ proceedings/2018/high-resolution-mapping-cross-over-events-cattleusing-ngs-data.pdf.

42. Druet T, Gautier M. A model-based approach to characterize individual inbreeding at both global and local genomic scales. Mol Ecol. 2017;26(20):5820-41. 
43. MacLeod IM, Larkin DM, Lewin HA, Hayes BJ, Goddard ME. Inferring demography from runs of homozygosity in whole-genome sequence, with correction for sequence errors. Mol Biol Evol. 2013;30(9):2209-23.

44. Druet T, Pérez-Pardal L, Charlier C, Gautier M. Identification of large selective sweeps associated with major genes in cattle. Anim Genet. 2013;44(6):758-62.

45. Gautier M, Faraut T, Moazami-Goudarzi K, Navratil V, Foglio M, Grohs C et al. Genetic and Haplotypic structure in 14 European and African cattle breeds. Genetics. 2007;177(2):1059-70.

46. Miar Y, Sargolzaei M, Schenkel FS. A comparison of different algorithms for phasing haplotypes using Holstein cattle genotypes and pedigree data. J Dairy Sci. 2017;100(4):2837-49.

47. Frioni N, Cavero D, Simianer H, Erbe M. Phasing quality assessment in a brown layer population through family- and population-based software. BMC Genet. 2019;20(1):57.

48. Money D, Wilson D, Jenko J, Whalen A, Thorn S, Gorjanc G, et al. Extending long-range phasing and haplotype library imputation algorithms to large and heterogeneous datasets. Genet Sel Evol. 2020;52(1):38.

49. Faux P, Druet T. A strategy to improve phasing of whole-genome sequenced individuals through integration of familial information from dense genotype panels. Genet Sel Evol. 2017;49(1):46.

50. Coop G, Wen X, Ober C, Pritchard JK, Przeworski M. High-resolution mapping of crossovers reveals extensive variation in fine-scale recombination patterns among humans. Science. 2008;319(5868):1395-8.

51. Harland C, Charlier C, Karim L, Cambisano N, Deckers M, Mni M, et al. Frequency of mosaicism points towards mutation-prone early cleavage cell divisions in cattle. bioRxiv. 2017:079863. https://doi.org/10.1101/079863.

52. Kadri NK, Harland C, Faux P, Cambisano N, Karim L, Coppieters W, et al. Coding and noncoding variants in HFM1, MLH3, MSH4, MSH5, RNF212, and RNF212B affect recombination rate in cattle. Genome Res. 2016;26(10):1323-32.

53. Lee Y-L, Takeda H, Moreira GCM, Karim L, Mullaart E, Coppieters W, et al. A 12 kb multi-allelic copy number variation encompassing a GC gene enhancer is associated with mastitis resistance in dairy cattle. PLoS Genet. 2021;17(7):e1009331.

54. Li H. Aligning sequence reads, clone sequences and assembly contigs with BWA-MEM. 2013; Available from: http://arxiv.org/abs/1303.3997.

55. Li H, Handsaker B, Wysoker A, Fennell T, Ruan J, Homer N, et al. The sequence alignment/map format and SAMtools. Bioinformatics. 2009;25(16):2078-9.

56. Tarasov A, Vilella AJ, Cuppen E, Nijman IJ, Prins P. Sambamba: fast processing of NGS alignment formats. Bioinformatics. 2015;31(12):2032-4.

57. Picard Toolkit [Internet]. Broad Institute, GitHub repository. 2019. Available from: https://broadinstitute.github.io/picard/

58. McKenna A, Hanna M, Banks E, Sivachenko A, Cibulskis K, Kernytsky A, et al. The genome analysis toolkit: a MapReduce framework for analyzing next-generation DNA sequencing data. Genome Res. 2010;20(9):1297-303.

59. DePristo MA, Banks E, Poplin R, Garimella KV, Maguire JR, Hartl C, et al. A framework for variation discovery and genotyping using next-generation DNA sequencing data. Nat Genet. 2011;43(5):491-8.

60. Van der Auwera GA, Carneiro MO, Hartl C, Poplin R, Del Angel G, LevyMoonshine A, et al. From FastQ data to high confidence variant calls: the genome analysis toolkit best practices pipeline. Curr Protoc Bioinformatics. 2013;43:11.10.1-11.10.33.

61. Nicolazzi EL, Picciolini M, Strozzi F, Schnabel RD, Lawley C, Pirani A, et al. SNPchiMp: a database to disentangle the SNPchip jungle in bovine livestock. BMC Genomics. 2014;15(1):123.

62. Layer RM, Chiang C, Quinlan AR, Hall IM. LUMPY: a probabilistic framework for structural variant discovery. Genome Biol. 2014;15(6):R84.

63. Browning BL, Tian $X$, Zhou $Y$, Browning SR. Fast two-stage phasing of large-scale sequence data. Am J Hum Genet. 2021;108(10):1880-90.

64. Marchini J, Cutler D, Patterson N, Stephens M, Eskin E, Halperin E, et al. A comparison of phasing algorithms for trios and unrelated individuals. Am J Hum Genet. 2006;78(3):437-50.

65. Duitama J, McEwen GK, Huebsch T, Palczewski S, Schulz S, Verstrepen K, et al. Fosmid-based whole genome haplotyping of a HapMap trio child: evaluation of single individual Haplotyping techniques. Nucleic Acids Res. 2012;40(5):2041-53.

\section{Publisher's Note}

Springer Nature remains neutral with regard to jurisdictional claims in published maps and institutional affiliations.
Ready to submit your research? Choose BMC and benefit from:

- fast, convenient online submission

- thorough peer review by experienced researchers in your field

- rapid publication on acceptance

- support for research data, including large and complex data types

- gold Open Access which fosters wider collaboration and increased citations

- maximum visibility for your research: over $100 \mathrm{M}$ website views per year

At BMC, research is always in progress.

Learn more biomedcentral.com/submissions 